\title{
Effects of Alanine Dipeptide on the Growth of an Ice Nucleus in Water*
}

\author{
Masahiro OHNO**, Kazuhisa IWASAKI** and Yoshimichi HAGIWARA***
}

\begin{abstract}
We have carried out a molecular dynamics simulation for the mixture of a cubic ice nucleus, supercooled water and a molecule of alanine dipeptide. The dipeptide molecule has been allocated near the nucleus surface. The dipeptide is found to approach the surface by the effect of hydrogen bond between two hydrophilic sites of the dipeptide and water molecules nearest to the liquid region in the ice nucleus. It is also found that the other hydrophilic sites far from the surface change their location with time due to the wobbling motion of the dipeptide molecule. This causes additional fluctuation of water molecules. These two sites and the hydrophobic site attenuate the approach of water molecules to the alanine dipeptide molecule or the ice surfaces.
\end{abstract}

Key Words: Molecular Dynamics Simulation, Ice Ih, Alanine Dipeptide, Hydrogen Bond

\section{Introduction}

Interest has been growing in the control of ice growth in ice-water mixture. The control of ice growth is one of the key technologies for the storage of food and the storage of internal organs in hospitals. Various methods have been proposed to improve this control process.

Among the methods, adding antifreeze protein (hereafter abbreviated as AFP) to the mixture has been focused on recently. Grandum et al. found from their observation by scanning tunneling microscope that the AFP type I formed the grooves at a specific location for a tiny ice crystal $^{(1)}$. They also carried out molecular simulation of AFP on the ice in the vacuum. They considered that the hydrophilic sites of AFP attached to the ice surface particularly normal to the $c$-axis of the crystal due to the hydrogen bond, thus the hydrophobic sites faces to water, which prevent freezing of water molecules.

Dalal et al. ${ }^{(2)}$ investigated the effect of AFP on the interface of water and ice crystal with a specific plane in detail by using a molecular dynamics simulation. They found that hydrogen bond is not the primary reason for

* Received 29th January, 2004 (No. 04-4042)

** Division of Mechanical and System Engineering, Kyoto Institute of Technology, Goshokaido-cho, Matsugasaki, Sakyo-ku, Kyoto 606-8585, Japan.

E-mail:kazu03@ipc.kit.ac.jp

*** Department of Mechanical and System Engineering, Kyoto Institute of Technology, Goshokaido-cho, Matsugasaki, Sakyo-ku, Kyoto 606-8585, Japan.

E-mail: yoshi@kit.ac.jp the interaction. However, the interaction between the specific surface of the ice crystal and AFP in a limited size of computation domain is not sufficient for discussing the effect of AFP in detail. Thus, the mechanism for interaction between an ice crystal and the AFP in water is still poorly understood.

This study aims at elucidating the interaction among the ice nucleus, the amino acids and surrounding water with a molecular dynamics simulation. The ice nucleus is useful not only for examining how it grows but also for reducing the effect of the boundary of computational domain on the ice-water interface. We use a molecule of alanine dipeptide (hereafter abbreviated as AD) for the hydrophobic amino acids. Approximately $65 \%$ of amino acids are alanines in AFP type I. In the first stage of our research, we have decided to ignore the effects of the other alanine and amino acids in AFP type I for simplicity. We focus solely on the interaction between one $\mathrm{AD}$ and water molecules.

\section{Nomenclature}

$D$ : self-diffusion function $\left[\mathrm{m}^{2}\right]$

$g$ : radial distribution function

$K$ : total energy [J]

$k_{B}$ : Boltzmann constant $\left[\mathrm{m}^{2}\right]$

$N$ : number of atoms or molecules

$q$ : electron charge

$r$ : coordinate of a molecule

$r$ : distance between two atoms or molecules [m]

$V$ : volume of computational domain $\left[\mathrm{m}^{3}\right]$ 
$v$ : translational velocity $[\mathrm{m} / \mathrm{s}]$

$\varepsilon, \sigma$ : parameters for potential $[\mathrm{J}],[\mathrm{m}]$

$\phi_{\mathrm{AD}}, \psi_{\mathrm{AD}}$ : dihedral angle of $\mathrm{AD}$ molecule [degrees]

$\omega$ : angular velocity [ $\mathrm{rad} / \mathrm{s}]$

\section{Simulation Method}

\subsection{Assumptions}

The classical molecular dynamics simulation was applied in this study. It was assumed that the number of molecules, the volume of computational domain and the total energy were kept constant (hereafter called the NVE ensemble) except for heating or energy removal procedures mentioned below. The molecules were assumed as rigid bodies. The net dipole moment, which was discussed by Hayward and Reimers ${ }^{(3)}$, was not evaluated in the present study. Then, the hydrogen bond is highlighted.

\subsection{Potential functions of water molecules}

The TIP4P potential ${ }^{(4)}$ was adopted for the potential function for the interaction between two water molecules. The potential function consists of the Coulomb potential and the Lennard-Jones potential.

The cutoff of the force acting on two distant molecules was done with the following methods: the cutoff radius for the Lennard-Jones potential and the Ewald $\operatorname{method}^{(5)}$ for the Coulomb potential. The cutoff radius was set at $2.245 \mathrm{~nm}$, which is equal to the dimension of the ice nucleus in the $x$-direction.

\subsection{Time integral}

The Newton-Euler equations for the translational and rotational motions were solved at each time step, and were integrated with the Gear algorithm ${ }^{(6)}$, a one-step predictorcorrector method. We adopted 5-value Gear algorithm in which the time derivatives up to the fifth order are considered for the second-order differential Newton-Euler equation for the translational motion. We also used 4-value Gear algorithm for the second-order differential equation for the rotation. All the computations were carried out with the time step of $0.5 \mathrm{fs}$.

\section{4 Estimation and adjustment of temperature}

The statistical temperature, $T$, was given with the law of equi-partition of energy by the total energy of the translational motion for all the molecules, $K_{T}$, and that of the rotational motion for the molecules, $K_{R}$. The temperature is written as follows:

$$
T=\frac{1}{2}\left(\frac{2}{3 k_{B} N} K_{T}+\frac{2}{3 k_{B} N} K_{R}\right),
$$

where $k_{B}$ is the Boltzmann constant, $N$ is the total number of molecules. The temperature scaling was done by changing the translational velocity, $v$, and the angular velocity, $\omega$, of each molecule with the following equation:

$$
\boldsymbol{v}_{i}^{(\text {new })}=\boldsymbol{v}_{i}^{(\text {old })} \sqrt{\frac{T_{p d}}{T_{0}}}, \omega_{i}^{(\text {new })}=\omega_{i}^{(\text {old })} \sqrt{\frac{T_{p d}}{T_{0}}},
$$

where $T_{0}$ is the present temperature and $T_{p d}$ is the predetermined temperature.

\section{Statistical Quantities}

\subsection{Self-diffusion function}

In order to judge whether the system is in a liquid state or a solid state, we used the self-diffusion function defined by the following equation:

$$
D(t)=\frac{1}{N} \sum_{i=1}^{N}\left(\boldsymbol{r}_{i}(t)-\boldsymbol{r}_{i}(0)\right)^{2},
$$

where $\boldsymbol{r}_{i}(t)$ is the coordinate of a molecule $i$ at time $t$. If the function increases with time, the system is in a liquid state. If the function does not change in time, the system remains in a solid state.

\subsection{Radial distribution function}

The radial distribution function of atoms for a specific atom was used for examining how many atoms exist in a region or how much they are arranged regularly. The function is expressed as follows ${ }^{(7)}$ :

$$
g(r)=\frac{V}{N^{2}}\left\langle\sum_{i} \sum_{j \neq i} \delta\left(\boldsymbol{r}_{i}-\boldsymbol{r}_{j}\right)\right\rangle,
$$

where $r=\left|\boldsymbol{r}_{i}-\boldsymbol{r}_{j}\right|$ is the distance between two atoms, $V$ is the volume of computational domain and $N$ is the number of atoms.

\section{Mixture of Ice and Water}

The result obtained in our previous study ${ }^{(8)}$ for the mixture of supercooled water with a cubic ice nucleus was used for the present computation. The outline how to obtain the mixture is described below.

\subsection{Formation of ice}

Sixty molecules were positioned so that the hexagonal structure was formed on a plane normal to the $z$-axis, and six planes were considered in a cubic domain for the initial condition. This arrangement of molecules is based on the ice Ih structure. The crystal can grow in the $x$ - and $y$-directions.

The rotational angle for each molecule at the initial state was given randomly. The Newton-Euler equations were solved with the proper constraint condition for the molecular displacement and the temperature scaling. It was confirmed from the self-diffusion function, the radial distribution function and snapshots that the ice Ih crystal state was sustained in the cases of temperatures lower than $265 \mathrm{~K}$.

\subsection{Production of ice-water mixture}

The new computational domain whose dimension was twice as large as that used above in the three directions was filled with eight identical ice crystals. The origin of the coordinate was set at the centre of this large computational domain. Only one of the crystals located near a lower corner of the domain $(-2.245 \mathrm{~nm} \leq x \leq 0 \mathrm{~nm}$, $-2.333 \mathrm{~nm} \leq y \leq 0 \mathrm{~nm},-2.200 \mathrm{~nm} \leq z \leq 0 \mathrm{~nm}$ ) was used 
as the ice nucleus, and the rest was melted by gradual heating and used as the liquid region. Through the heating, the temperature scaling was applied intermittently for the translational velocity of each molecule in the liquid region so that the water temperature reached a predetermined temperature. The heating continued until the predetermined temperature was equal to a target temperature. The total energy input was about $90 \%$ of the latent heat.

\subsection{Mixture of ice and supercooled water}

We removed the energy from the liquid region gradually in order to obtain the supercooled water. A similar procedure to that used for the melting of ice mentioned above was applied for the energy removal procedure: Through the cooling, the temperature scaling was applied intermittently for the translational velocity of each molecule in the liquid region so that the water temperature reached a predetermined temperature. The removal of energy continued until the predetermined temperature was equal to the target temperature of $240 \mathrm{~K}$. The total energy removed was $161 \mathrm{~kJ} / \mathrm{kg}$. This is approximately $50 \%$ of the latent heat.

\section{AD Molecule}

\subsection{Conformation parameters}

Figure 1 shows the AD molecule. The OPLS parameters obtained by Jorgensen and Tirado-Rives ${ }^{(9)}$ were adopted to the potential parameters for each site of the $\mathrm{AD}$ molecule. The parameters are summarized in Table 1. The center of mass of the molecule was close to the $\alpha$-Carbon, $\mathrm{C}^{\alpha}$ (Atom \#; 6, Atom type; $\mathrm{CH}$ ).

The dihedral angle around the $\mathrm{C}^{\alpha}-\mathrm{N}$ bond, $\phi_{\mathrm{AD}}$, and that around the $\mathrm{C}^{\alpha}-\mathrm{C}$ bond, $\psi_{\mathrm{AD}}$, were determined so that the $\mathrm{AD}$ molecule took the conformation called $\alpha$-helix. This is because the alanines usually take the $\alpha$-helix in proteins and the AFP type I has a helical structure. According to the Ramanchandran plots and the result obtained by Gould et al. ${ }^{(10)}$, we set $\phi_{\mathrm{AD}}=-60.7$ degrees and $\psi_{\mathrm{AD}}=-40.7$ degrees.

\subsection{Introduction of AD molecule into water}

After the energy removal procedure, the interaction between the ice nucleus and the supercooled water was started. The initial state for the present simulation was the result at $100 \mathrm{ps}$ after the initiation of the interaction between ice nucleus and the supercooled water. The average energies and the average temperature for $5 \mathrm{ps}$ just before the initial state are shown in Table 2.

The liquid region was divided into two regions: the near-ice region and the far-ice region. The near-ice region covered the whole surface of the ice nucleus. The thickness of the region was $2.5 \mathrm{~nm}$.

The AD molecule was introduced into a specific part of the near-ice region, which is adjacent to one of the surface normal to the $x$-axis. A number of water molecules were eliminated from a spherical domain in the near-ice

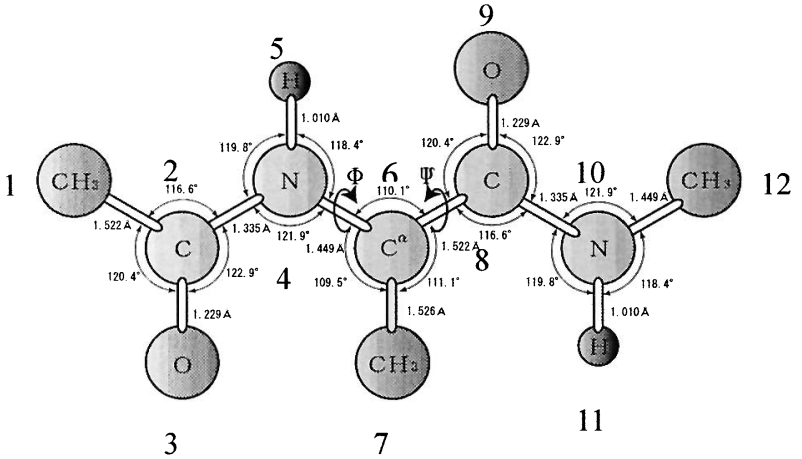

Fig. 1 AD molecule

Table 1 Parameters for AD molecule

\begin{tabular}{|c|c|c|c|c|}
\hline Atom \# & Atom type & $\mathrm{Q}$ & $\sigma(\mathrm{nm})$ & $\varepsilon(\mathrm{kJ} / \mathrm{mol})$ \\
\hline \multicolumn{5}{|c|}{ Hydrophobic sites } \\
\hline 1,7 & C31, C32 & 0.0 & 0.391 & 0.670 \\
\hline 12 & $\mathrm{JW}$ & 0.2 & 0.38 & 0.712 \\
\hline \multicolumn{5}{|c|}{ Hydrophilic sites } \\
\hline 2,8 & $\mathrm{C} 1, \mathrm{C} 2$ & 0.5 & 0.375 & 0.440 \\
\hline 3,9 & Oc1, Oc2 & -0.5 & 0.296 & 0.879 \\
\hline 4,10 & $\mathrm{~N} 1, \mathrm{~N} 2$ & -0.57 & 0.325 & 0.712 \\
\hline 5,11 & $\mathrm{Hn} 1, \mathrm{Hn} 2$ & 0.37 & 0 & 0 \\
\hline 6 & $\mathrm{CH}$ & 0.2 & 0.38 & 0.335 \\
\hline
\end{tabular}

Table 2 Mean values of potential energy, translational temperature and rotational temperature for ice and water

\begin{tabular}{lll}
\hline & \multicolumn{2}{l}{ Ice nucleus Water } \\
\hline Potential energy $(\mathrm{kJ} / \mathrm{mol})$ & -48.2 & -45.1 \\
Translational kinetic energy $(\mathrm{kJ} / \mathrm{mol})$ & 3.02 & 3.01 \\
Translational temperature $(\mathrm{K})$ & 242.3 & 241.5 \\
Rotational kinetic energy $(\mathrm{kJ} / \mathrm{mol})$ & 3.11 & 3.00 \\
Rotational temperature $(\mathrm{K})$ & 249.2 & 240.4 \\
\hline
\end{tabular}

region. The radius of domain was set equal to the length of the longer axis of the AD molecule. The total number of water molecules was 21 . Figure 2 shows the initial location of the $\mathrm{AD}$ molecule. The initial coordinates of Hn1 site were $(0.491 \mathrm{~nm},-1.054 \mathrm{~nm},-1.016 \mathrm{~nm})$ and that of Oc2 site were $(0.499 \mathrm{~nm},-1.272 \mathrm{~nm},-1.212 \mathrm{~nm})$. The translation and rotation velocities of the molecule were set equal to zero. The computational condition is summarized in Table 3.

The temperature scaling was carried out after the AD molecule was introduced in order to attenuate an unrealistic rapid increase in the energy due to the inconsistency of the arrangement of the molecules. The scaling procedure was summarized in Table 4 . For the first $5 \mathrm{ps}$, the molecules in ice were fixed and the displacement of the $\mathrm{AD}$ molecule was reduced to $0.1 \%$ of its predicted value. The temperature scaling was carried out at each time step. For the next 5 ps $(5-10 \mathrm{ps})$, the displacements of the molecules in ice and the AD molecule were reduced to $0.1 \%$ of their predicted value. The scaling was carried out at each time step. For the third 5 ps (10-15 ps), only 
Table 3 Computational condition

\begin{tabular}{lc}
\hline Domain $\left(\mathrm{nm}^{3}\right)$ & $4.491 \times 4.667 \times 4.400$ \\
Time step (fs) & 0.5 \\
Number of molecules in ice nucleus & 360 \\
Number of molecules in liquid region & 2499 \\
Number of AD molecule & 1 \\
Initial temperature of ice nucleus $(\mathrm{K})$ & 245.1 \\
Initial temperature of liquid region $(\mathrm{K})$ & 239.3 \\
Predetermined temperature of ice nucleus $(\mathrm{K})$ & 245.8 \\
Predetermined temp. of liquid region and AD(K) & 241.0 \\
\hline
\end{tabular}

Table 4 Scaling procedure

\begin{tabular}{lllll}
\hline Period & Water & Ice nucleus & AD & Scaling interval \\
\hline $0-5(\mathrm{ps})$ & Free & Fix & $0.1 \%$ & $0.5(\mathrm{fs})$ \\
$5-10(\mathrm{ps})$ & Free & $0.1 \%$ & $0.1 \%$ & $0.5(\mathrm{fs})$ \\
$10-15(\mathrm{ps})$ & Free & Free & Free & $0.5(\mathrm{fs})$ \\
$15-95(\mathrm{ps})$ & Free & Free & Free & - \\
\hline
\end{tabular}
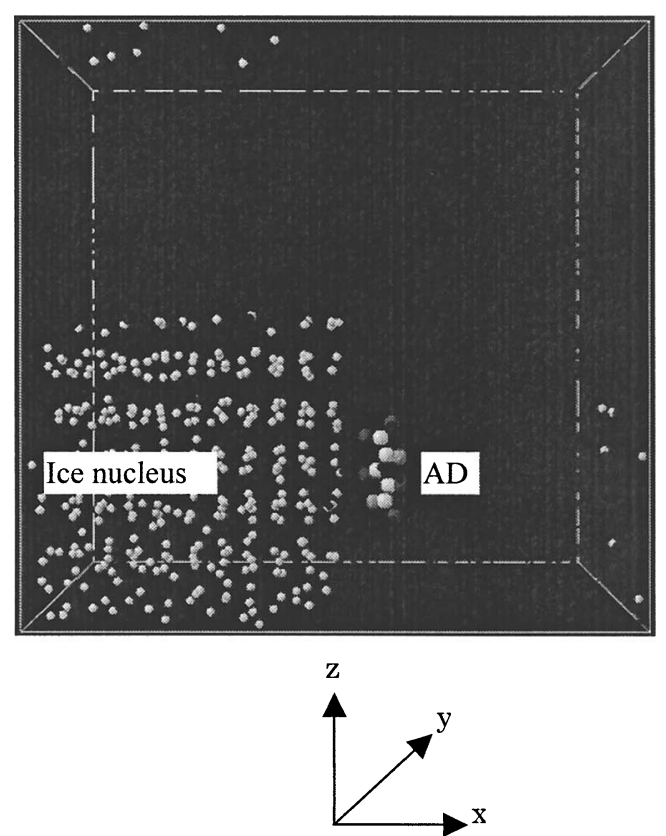

Fig. 2 Snapshot for the initial condition

the scaling was carried out at each time step. The temperature scaling was terminated at $15 \mathrm{ps}$.

One of the present authors carried out the computation without the $\mathrm{AD}$ molecule in order to obtain reference data for the radial distribution function of oxygen atoms $^{(11)}$.

\section{Results and Discussion}

\subsection{Total energy of system}

It was confirmed that the total potential energy and the total kinetic energy were kept constant throughout the computation $^{(11)}$. Thus, the present computation based on the micro-canonical $(N V E)$ ensemble was validated. Furthermore, the system was confirmed to be stable.

\subsection{Interaction between ice nucleus and AD molecule}

7.2.1 Radial distribution function for sites Figure 3 indicates the radial distribution functions for the oxygen atoms $(\mathrm{Ow})$ and the hydrogen atoms $(\mathrm{Hw})$ of water molecules for seven sites of the AD molecules. The first peak and the first valley in the function for $\mathrm{Ow}$ are shifted noticeably from that for $\mathrm{Hw}$ in the cases of polar sites (particularly in the case of Oc groups). On the other hand, the shift is not noticeable in the case of nonpolar methyl groups. These reflect the existence of hydrogen bond between the polar AD sites and the water molecules. Since the carbonyl oxygens carry negative charges, Hw tends to come closer compared with Ow. Since the amide hydrogens carry positive charges, Ow tends to come closer compared with Hw. These results are in agreement with those obtained by Kalko et al. ${ }^{(12)}$ However, the first peak is not clear for Hn sites. This is due to the TIP4P model for water molecules. In this model, a positive charge is given at each hydrogen atom, while a negative charge is not allocated at the oxygen atom but just outside of the oxygen atom (the hydrogen side).

7.2.2 Motion of AD molecule Figure 4 shows the time changes in the $x$ coordinate of each site of the AD molecule. All these sites are found to be transported to the negative direction of the $x$-axis. This shows that the $\mathrm{AD}$ molecule approached the ice nucleus. For the period of 20-55 ps, the $x$ coordinates of Hn1, C31 and C32 sites decreased rapidly, while those of $\mathrm{Hn} 2$, Oc2 and JW sites decreased gradual. This is due to the rotational motion of the $\mathrm{AD}$ molecule for the period. This was confirmed by the result that the rotational kinetic energy for the AD molecule took higher values than those in the other pe$\operatorname{riod}^{(11)}$.

After $55 \mathrm{ps}$, the time changes in the $x$ coordinate for all the sites are more or less similar. This is due to the translational motion of the AD molecule for the period. This was confirmed by the result that the translational kinetic energy for the AD molecule was more predominant than the rotational kinetic energy in this period ${ }^{(11)}$. The translational motion of the AD molecule was observed from the snapshots during the same period. The Oc2 site and Hn1 site were basically the closest to the crystal for the period. This was also observed in the case of different initial orientation of the AD molecule ${ }^{(13)}$.

After $95 \mathrm{ps}$, the $x$ coordinate for each site fluctuated at a certain value.

7.2.3 Mechanism of ice-AD interaction Figure 5 depicts a typical example for the number of water molecules, $N_{w}$, inside the spherical shell for five sites as a function of the shell radius $r_{s}$. The shaded area shows the number of water molecules in the ice nucleus. There is no water molecule inside the spherical shell of $r_{s}=0.26 \mathrm{~nm}$ for Hn1 site. This radius is the radius for the first hy- 


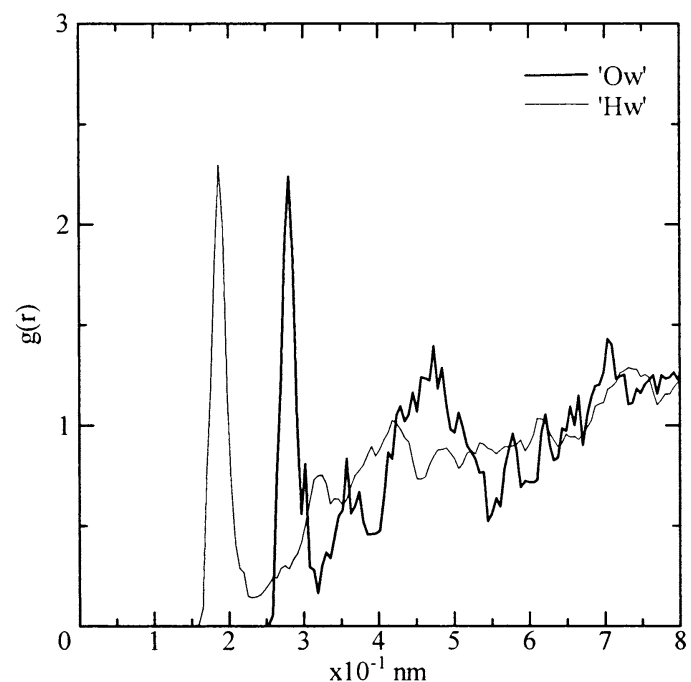

(a) Oc group

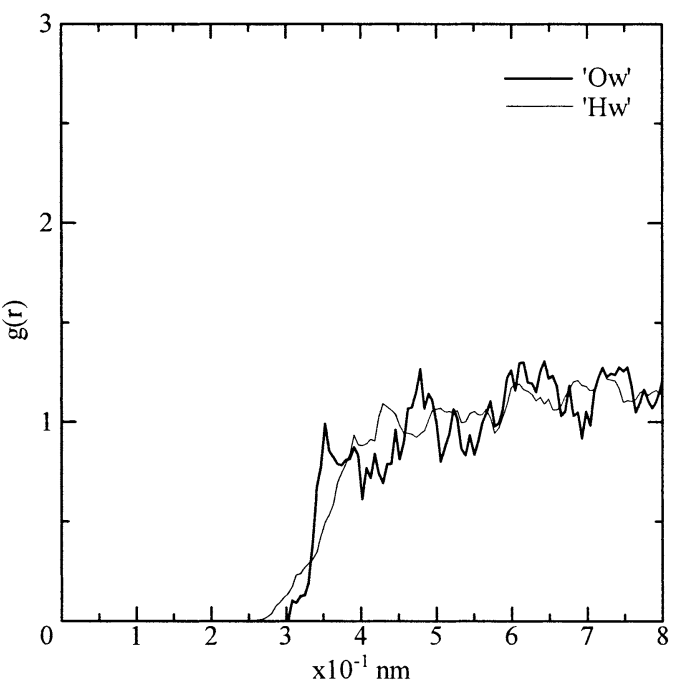

(b) Me group

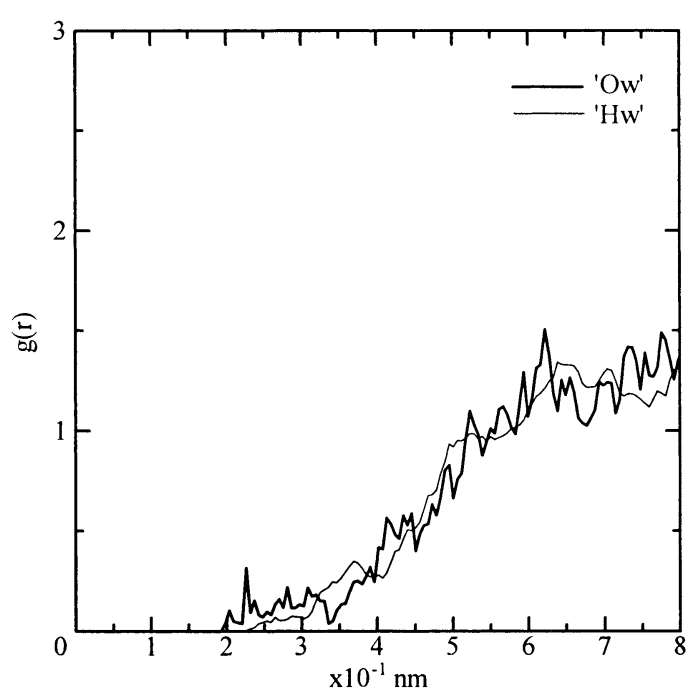

(c) Hn group

Fig. 3 Radial distribution function for sites of $\mathrm{AD}$ molecules

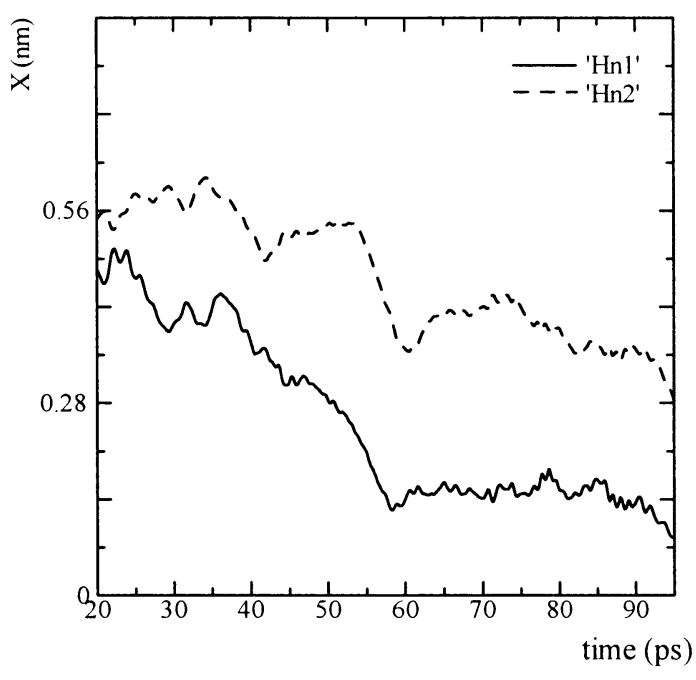

(a) Hn group

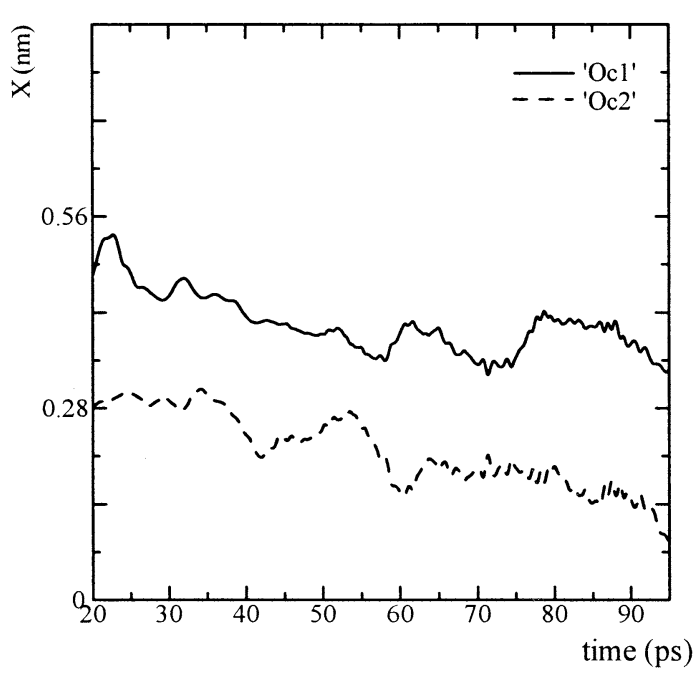

(b) Oc group

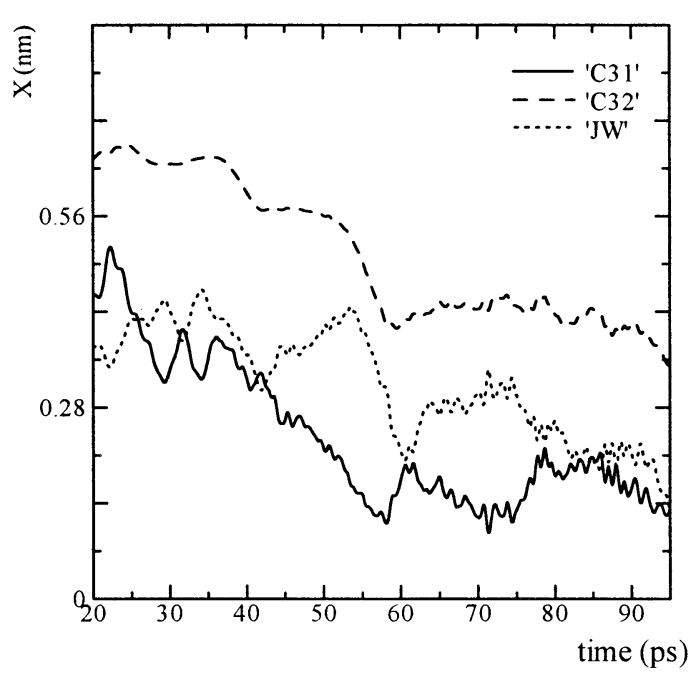

(c) Me group

Fig. 4 Time changes in the $x$-coordinate of sites 


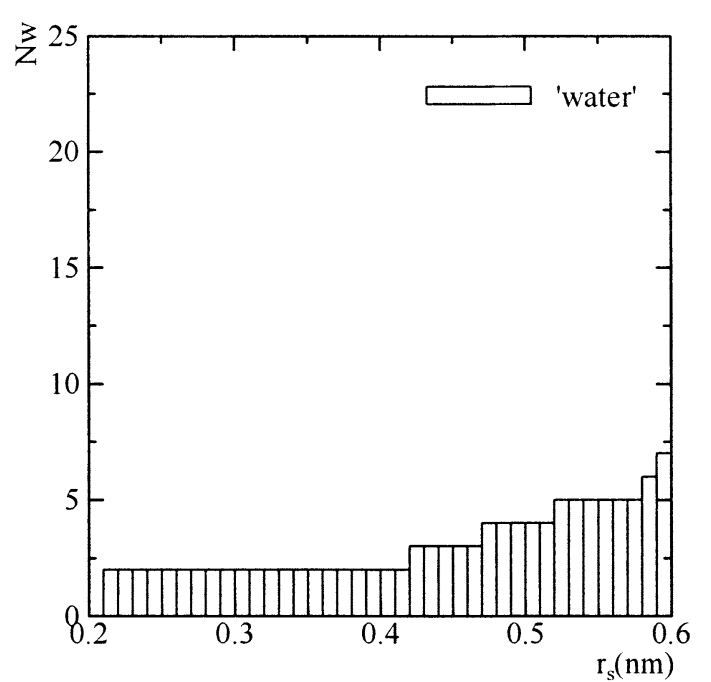

(a) Oc1

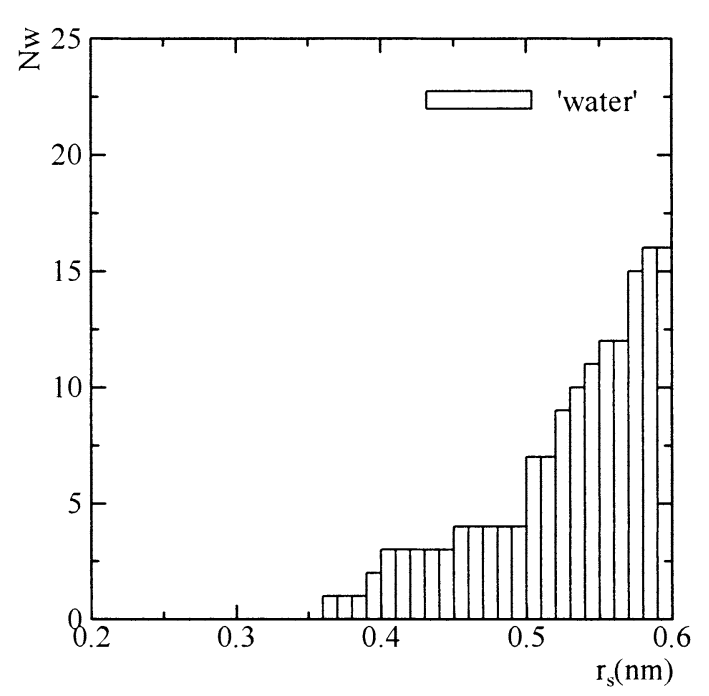

(b) $\mathrm{C} 32$

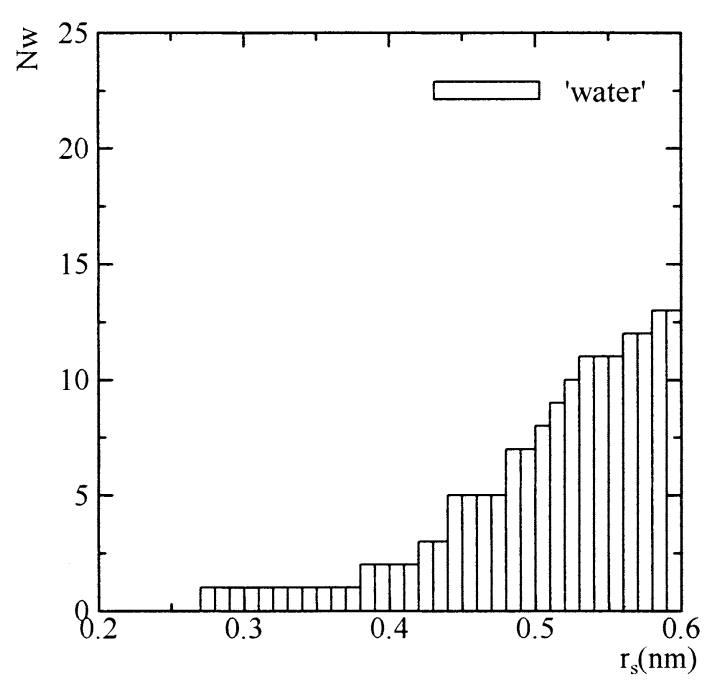

(c) $\mathrm{Hn} 2$

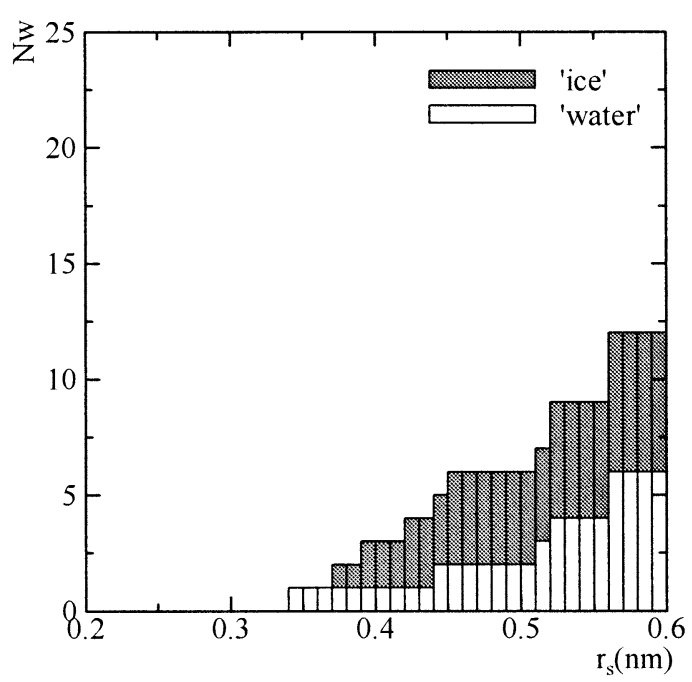

(d) Hn1

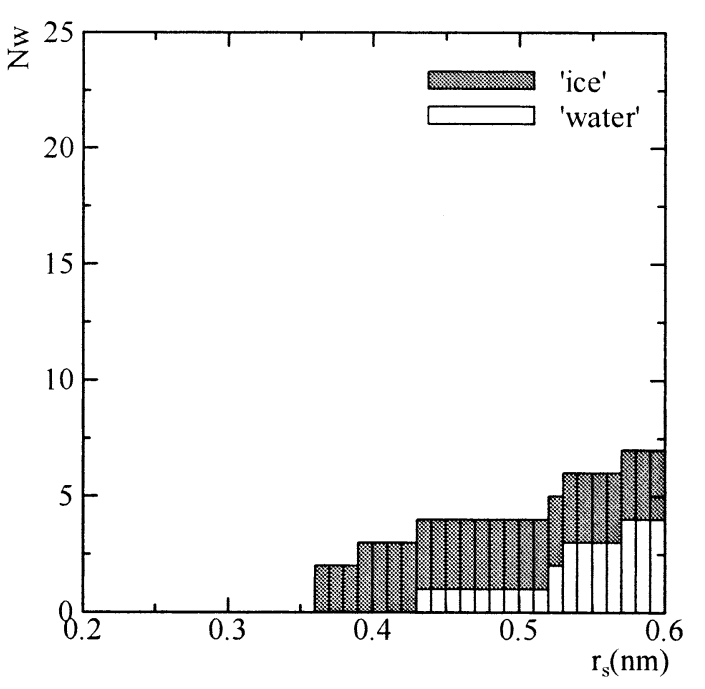

(e) Oc2

Fig. 5 Number of water molecules inside spherical shell

dration shell for Hn sites obtained by Kalko et al. ${ }^{(12)}$ One water molecule in the ice nucleus was inside the shell in the other time. Although three water molecules in the ice nucleus are inside the first hydration shell for Oc2 site $\left(r_{s}=0.40 \mathrm{~nm}\right)$, there is no water molecule in the liquid region inside the shell. These indicate that Hn1 and Oc2 interact directly with the water molecules in the ice nucleus.

The site distance between $\mathrm{Hn} 1$ and Oc2 is $0.30 \mathrm{~nm}$. The distance between the oxygen atom of a water molecule and the hydrogen atom of another water molecule is $0.17 \mathrm{~nm}$. Judging from these values, Hn1 and Oc2 may cause the hydrogen bond to the water molecule nearest to the liquid region in the ice nucleus. The hydrogen bond of H-O $\cdots H n 1$ takes approximately 120 degrees, which can be realized by the site. The hydrogen bond of O-H...Oc2 takes approximately 180 degrees, which can- 


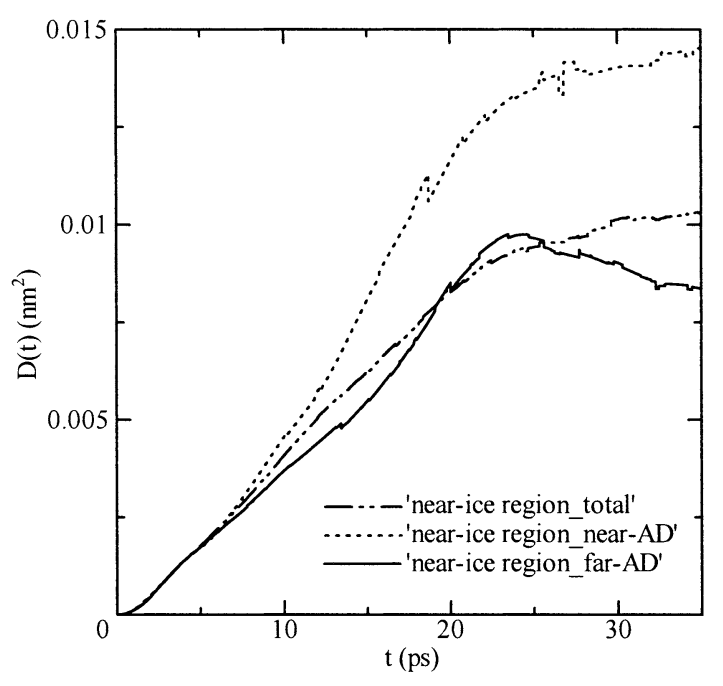

Fig. 6 Self-diffusion functions (60-95 ps)

not be realized by the AD molecule outside of the ice nucleus. Thus, the latter hydrogen bond is weaker than the former one. This allows the fluctuation of the location of AD molecule.

\subsection{Interaction between AD molecule and water}

7.3.1 Self-diffusion of water molecules Figure 6 shows the self-diffusion function of water molecules in the period of $60-95 \mathrm{ps}$. This function was calculated only for the water molecules in the specific regions by using a conditional sampling. The diffusion of molecules in the region of near ice and far from the AD molecule is drawn with the solid line. This line resembles that of the molecules in the whole near-ice region drawn with the dash-dotted line. On the other hand, the self-diffusion function of water molecules in the region of near ice and near the $\mathrm{AD}$ molecule drawn with the dotted line takes much higher values than those of the other cases. Therefore, the AD molecule enhances the diffusion of the molecules of supercooled water. This suggests that the AD molecule attenuate the solidification of water molecules.

\subsubsection{Radial distribution function for oxygen} atoms Figure 7 demonstrates the radial distribution functions for the oxygen atoms of water molecules in near$\mathrm{AD}$ region and far-AD region in the two periods. The values of $g(r)$ were calculated only for the oxygen atoms in the specific regions by using a conditional sampling. Since these regions were not so thick, the number of the oxygen atoms decreased with an increase in $r$, particularly in the region where $r$ was longer than the half of the region thickness $0.374 \mathrm{~nm}$. Therefore, we focus on the region of $r<0.374 \mathrm{~nm}$.

The first peak and the first valley for RDF in the case of near-AD region are found to be slightly higher than those in the case of far-AD region in both periods. In Ref. (11), the peak and the valley of RDF for water molecules in liquid region were higher than those in the ice

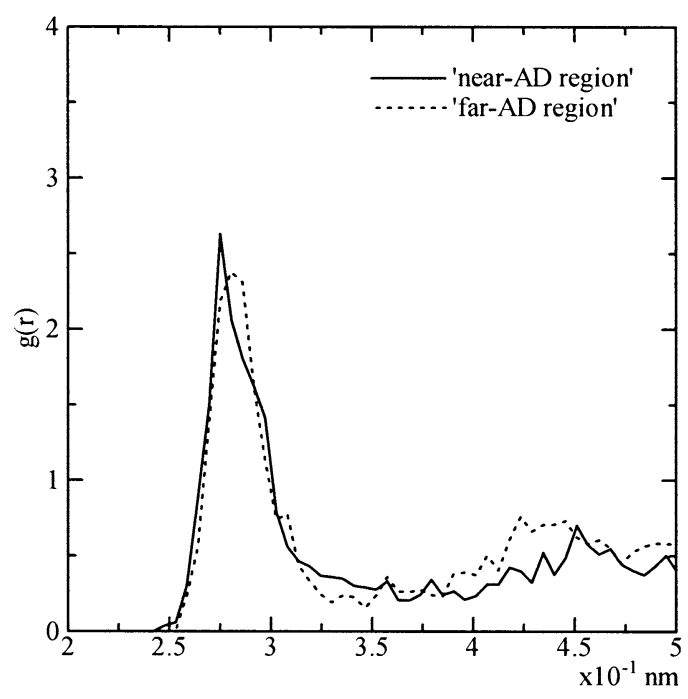

(a) $20-25 \mathrm{ps}$

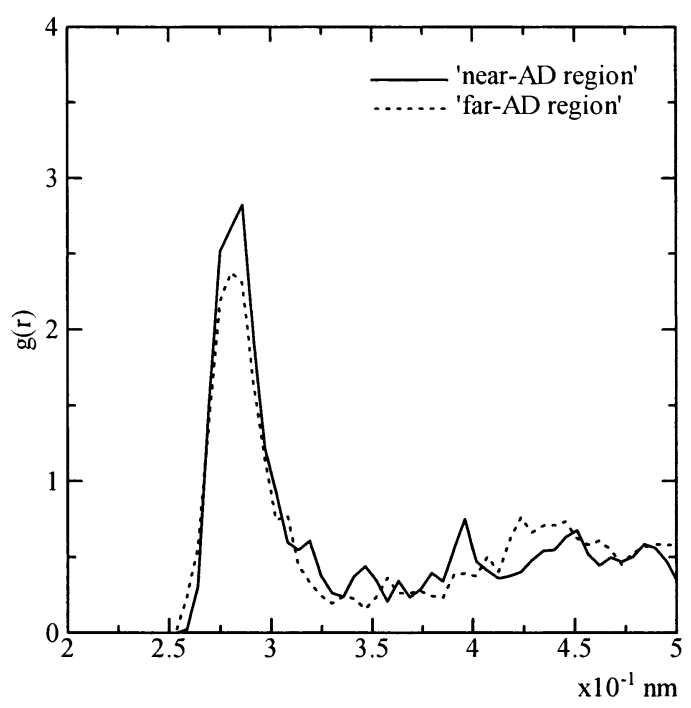

(b) $90-95 \mathrm{ps}$

Fig. 7 O-O radial distribution functions

in the case without the AD molecule. Therefore, the water molecules in the near-AD region behave as liquid more than those in the far-AD region do. This result also shows an attenuation of the solidification of water molecules by the AD molecule.

7.3.3 Mechanism of AD-water interaction The locations of hydrophilic sites of Oc1 and Hn2 far from the ice nucleus changed their positions with time by the wobbling motion of the AD molecule mainly on $(y$, z)-plane. We estimated the amplitude and period of this motion from the animation of the whole computational results. The amplitude was approximately $0.1 \mathrm{~nm}$, and the period was approximately $8 \mathrm{ps}$. The motion of $\mathrm{AD}$ molecule may cause additional fluctuation of water molecules near Oc1 and Hn2. This additional fluctuation arrests the approach of water molecules in the liquid phase to the water molecules on the ice surface or the AD molecule. Furthermore, no water molecule came close 
to the hydrophobic site of C32, as shown in Fig. 5 (b). Therefore, all the sites of AD molecule far from the ice nucleus attenuated the approach of water molecules to the $\mathrm{AD}$ molecule or the ice surfaces.

\section{Conclusions}

The molecular dynamics simulation was carried out in order to understand the interaction among the AD molecule, the water molecules in the ice nucleus and those in the liquid region. The main conclusions obtained are as follows.

(1) The AD molecule moved towards the surface of the ice nucleus so that the weak hydrogen bond between the oxygen atom of water molecule closest to the liquid region in the ice and $\mathrm{Hn} 1$ site of the $\mathrm{AD}$ molecule and the weak hydrogen bond between the hydrogen atom of the water molecule and $\mathrm{Oc} 2$ site of the $\mathrm{AD}$ molecule can be realized. The distance between $\mathrm{Hn} 1$ and Oc2 is shorter than the distance between $\mathrm{Hw}$ and $\mathrm{Ow}$ of the water molecule. The hydrogen bond of Ow..Hn 1 is stronger than that of Hw...Oc2. This allows the fluctuation of the location of AD molecule.

(2) Due to the wobbling motion of the AD molecule, the hydrophilic sites of Oc1 and Hn2 changed their location with time. This causes an additional fluctuation of water molecules. These two sites and the hydrophobic site of C32 far from the ice nucleus attenuated the approach of water molecules to the AD molecule or the ice surfaces.

\section{References}

(1) Grandum, S., Yabe, A., Nakagomi, K., Tanaka, M., Takemura, F., Kobayashi, Y. and Frivik, P., Microscale Analysis of Crystals in Ice Slurry Made from an Antifreeze Protein Solution, Trans. Jpn. Soc. Mech. Eng., (in Japanese), B, Vol.63 (1997), pp.1029-1036.

( 2 ) Dalal, P., Knickelbein, J., Haymet, A.D.J., Sönnichsen, F.D. and Madura, J.D., Hydrogen Bond Analysis of
Type 1 Antifreeze Protein in Water and the Ice/Water Interface, Phys. Chem. Comm., Vol.4 (2001), pp.3240.

( 3 ) Hayward, J.A. and Reimers, R., Unit Cells for the Simulation of Hexagonal Ice, J. Chem. Phys., Vol.106 (1997), pp.1518-1529.

( 4 ) Jorgensen, W.J., Chandrasekhar, J., Madura, J.D., Impey, R.W. and Klein, M.L., Comparison of Simple Potential Functions for Simulating Liquid Water, J. Chem. Phys., Vol.79 (1983), pp.926-935.

( 5 ) Frenkel, D. and Smit, B., Understanding Molecular Simulation, (1996), Academic Press, San Diego.

( 6 ) Gear, C.W., Numerical Initial Value Problems in Ordinary Differential Equations, (1971), Prentice-Hall, Englewood Cliffs.

( 7 ) Allen, M.P. and Tildesley, D.J., Computer Simulation of Liquid, (1987), Oxford Science, Oxford.

( 8 ) Yokoyama, T. and Hagiwara, Y., Molecular Dynamics Simulation for the Mixture of Water and an Ice $\mathrm{Nu}-$ cleus, Molecular Simulation, Vol.29 (2003), pp.235248.

( 9 ) Jorgensen, W.J. and Tirado-Rives, J., The OPLS Potential Functions for Proteins: Energy Minimizations for Crystals of Cyclic Peptides and Crambin, J. Am. Chem. Soc., Vol.110 (1988), pp.1657-1666.

(10) Gould, I.R., Cornell, W.D. and Hillier, I.H., A Quantum Mechanical Investigation of the Conformational Energetics of the Alanine and Glycine Dipeptides in the Gas Phase and in Aqueous Solution, J. Am. Chem. Soc., Vol.116 (1994), pp.9250-9256.

(11) Ohno, M., Molecular Dynamics Simulation for the System of Ice Nucleus and Water with Alanine Dipeptide, (in Japanese), M. Eng. Thesis, Kyoto Institute of Technology, (2003).

(12) Kalko, S.G., Guàrdia, E. and Padró, J.A., Molecular Dynamics Simulation of the Hydration of the Alanine Dipeptide, J. Phys. Chem., B, Vol.103 (1999), pp.3935-3941.

(13) Iwasaki, K. and Hagiwara, Y., Molecular Simulation, Vol.30, No.8 (2004), pp.487-500. 